\title{
Giant pressure-enhancement of multiferroicity in $\mathrm{CuBr}_{2}$
}

\author{
J. S. Zhang, ${ }^{1,2, *}$ Yiqi Xie,,${ }^{3, *}$ X. Q. Liu, ${ }^{3}$ A. Razpopov, ${ }^{4}$ V. Borisov, ${ }^{4}$ C. Wang, ${ }^{3}$ J. P. Sun $\odot,{ }^{5,6}$ Y. Cui, ${ }^{2}$ J. C. Wang, ${ }^{2}$ \\ X. Ren, ${ }^{3}$ Hongshan Deng, ${ }^{7}$ Xia Yin, ${ }^{7}$ Yang Ding, ${ }^{7}$ Yuan Li,${ }^{3,8, \dagger}$ J. G. Cheng $\odot,{ }^{5,6,9,}$ Ji Feng, ${ }^{3,8}$ \\ R. Valentí, ${ }^{4}$ B. Normand, ${ }^{10}$ and Weiqiang $\mathrm{Yu}^{2,}$, \\ ${ }^{1}$ Mathematics and Physics Department, North China Electric Power University, Beijing 102206, China \\ ${ }^{2}$ Department of Physics, and Beijing Key Laboratory of Opto-electronic Functional Materials \& Micro-nano Devices, \\ Renmin University of China, Beijing 100872, China \\ ${ }^{3}$ International Center for Quantum Materials, School of Physics, Peking University, Beijing 100871, China \\ ${ }^{4}$ Institut für Theoretische Physik, Goethe-Universität Frankfurt, 60438 Frankfurt am Main, Germany \\ ${ }^{5}$ Beijing National Laboratory for Condensed Matter Physics and Institute of Physics, Chinese Academy of Sciences, Beijing 100190, China \\ ${ }^{6}$ School of Physical Sciences, University of Chinese Academy of Sciences, Beijing 100190, China \\ ${ }^{7}$ Center for High Pressure Science and Technology Advanced Research (HPSTAR), Beijing 100193, China \\ ${ }^{8}$ Collaborative Innovation Center of Quantum Matter, Beijing 100871, China \\ ${ }^{9}$ Songshan Lake Materials Laboratory, Dongguan, Guangdong 523808, China \\ ${ }^{10}$ Neutrons and Muons Research Division, Paul Scherrer Institute, CH-5232 Villigen-PSI, Switzerland
}

(Received 11 November 2019; accepted 10 January 2020; published 10 February 2020)

\begin{abstract}
Type-II multiferroic materials, in which ferroelectric polarization is induced by inversion nonsymmetric magnetic order, promise new and highly efficient multifunctional applications based on the mutual control of magnetic and electric properties. Although this phenomenon has to date been limited to low temperatures, here we report a giant pressure dependence of the multiferroic critical temperature in $\mathrm{CuBr}_{2}$. At $4.5 \mathrm{GPa}, T_{\mathrm{C}}$ is enhanced from 73.5 to $162 \mathrm{~K}$, to our knowledge the highest value yet reported for a nonoxide type-II multiferroic. This growth shows no sign of saturating and the dielectric loss remains small under these high pressures. We establish the structure under pressure and demonstrate a $60 \%$ increase in the two-magnon Raman energy scale up to $3.6 \mathrm{GPa}$. First-principles structural and magnetic energy calculations provide a quantitative explanation in terms of dramatically pressure-enhanced interactions between $\mathrm{CuBr}_{2}$ chains. These large, pressure-tuned magnetic interactions motivate structural control in cuprous halides as a route to applied high-temperature multiferroicity.
\end{abstract}

DOI: 10.1103/PhysRevResearch.2.013144

\section{INTRODUCTION}

The search for application-suitable multiferroics [1-3] has advanced significantly over the last decade in both type-I and type-II materials [4-9]. Type-I multiferroics [10] have independent magnetic and ferroelectric transitions [11,12], meaning that even when both transition temperatures are high, the magnetoelectric coupling, and hence the scope for mutual control, is usually weak. The physics of most type-II multiferroics [10,13-15] involves frustrating magnetic interactions that give rise to a spiral magnetic order [16], which immediately generates a ferroelectric polarization by the inverse Dzyaloshinskii-Moriya mechanism [17-21]. However, an intrinsic drawback of magnetic frustration is that it

\footnotetext{
*These authors contributed equally to this work.

†yuan.li@pku.edu.cn

¥jgcheng@iphy.ac.cn

$\S_{\text {wqyu_phy@ruc.edu.cn }}$
}

Published by the American Physical Society under the terms of the Creative Commons Attribution 4.0 International license. Further distribution of this work must maintain attribution to the author(s) and the published article's title, journal citation, and DOI. suppresses the onset of long-range order, and hence most currently available type-II multiferroics operate only at low temperatures [14].

A generic route to higher operating temperatures in type-II multiferroics is to increase the strength of the magnetic interactions. This can, in principle, be achieved through structural alterations, for which perhaps the cleanest method is an applied pressure [22-26]. Pressure, broadly construed to include chemical pressure and substrate pressure, acts to increase electronic hybridization without introducing disorder. In the most minimal model for a magnetic insulator, the antiferromagnetic (AF) exchange interaction is given by $J=4 t^{2} / U$, where $t$ is the orbital hybridization and $U$ is the on-site Coulomb repulsion. However, excessive $t$ risks driving the system metallic, thus losing its magnetic and ferroelectric properties. The most scope for achieving large $J$ values is offered by large initial values of both $t$ and $U$, making the spin- $1 / 2 \mathrm{Cu}^{2+}$ ion particularly promising in view of its often strong on-site correlations and significant orbital hybridization with ligands. It is not a coincidence that complex copper oxides become high-temperature superconductors after charge-carriers are introduced into the Mott-insulating parent compounds [27], or that $\mathrm{CuO}$ is a type-II multiferroic with the co-highest transition temperature $\left(T_{\mathrm{C}} \simeq 230 \mathrm{~K}\right)$ known to date [28]. 


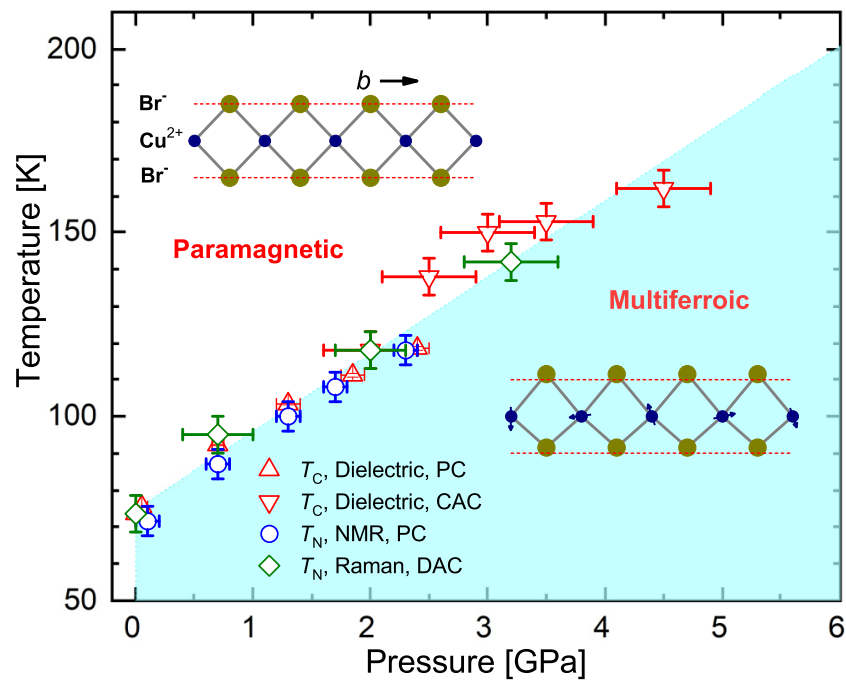

FIG. 1. Phase diagram of $\mathrm{CuBr}_{2}: T_{\mathrm{C}}(P)$ and $T_{\mathrm{N}}(P)$ determined from dielectric, NMR, and Raman scattering measurements. A piston cell (PC), a cubic anvil cell (CAC), and a diamond anvil cell (DAC) were used for different pressure ranges and measurements as specified. The upper inset shows a schematic representation of the chain structure in the high-temperature paramagnetic phase; the lower shows a representation of the chain structure and magnetism in the low-temperature $\left[T<T_{\mathrm{N}}(P)\right]$ multiferroic phase, where the magnetic order (arrows) is helical in the chain direction, breaking inversion symmetry, and the ferroelectricity is caused by small displacements of the $\mathrm{Br}^{-}$ions (exaggerated for illustration).

$\mathrm{CuBr}_{2}$ is a nonoxide type-II multiferroic material with a $\mathrm{CdI}_{2}$-type monoclinic structure [29]. The structural units are $\mathrm{CuBr}_{4}$ squares, which form edge-sharing chains in the $b$ direction (see insets of Fig. 1). These chains have a $C$-centered stacking in the $a$ direction and coincidentally form nearly coplanar units in the $b(a+c)$ plane. Early first-principles calculations [30] of the magnetic interactions indicated that the dominant coupling $\left[J_{5}\right.$ in Fig. 6(a) $]$ is that between nextnearest-neighbor $\mathrm{Cu}^{2+}$ ions within the chains, which is AF. Other strong interactions are expected to be the ferromagnetic (FM) nearest-neighbor in-chain bond $\left(J_{1}\right)$, and the AF coupling between sites in coplanar chains $\left(J_{7}\right)$; additional weak interactions were suggested to be responsible for the formation of long-ranged three-dimensional (3D) magnetic order. Frustration between $J_{1}$ and $J_{5}$ suggests spiral order along the chains, with the spin rotation angle given classically by $\theta=\cos ^{-1}\left(-J_{1} / 4 J_{5}\right)$, which approaches $90^{\circ}$ when $J_{5}$ significantly exceeds $\left|J_{1}\right|$. At ambient pressure, a spiral magnetic order does indeed develop below $T_{\mathrm{N}}=73.5 \mathrm{~K}$, with an incommensurate propagation wave vector $(1,0.2350$, $0.5)[29,30]$ fully consistent with the expected $\theta$. Spontaneous electric polarization is detected immediately below $T_{\mathrm{N}}$, defining a rather high ferroelectric transition temperature [29]; while $T_{\mathrm{C}}=T_{\mathrm{N}}$ by definition in a type-II multiferroic, below we distinguish between the two according to our method of experimental detection. Similar properties have been found in the isostructural compound $\mathrm{CuCl}_{2}$, albeit at considerably lower temperatures [31].

Here we report our investigation of ferroelectricity and magnetism in $\mathrm{CuBr}_{2}$ under hydrostatic pressure. By combined dielectric-constant, nuclear magnetic resonance (NMR), Raman-scattering, and $\mathrm{x}$-ray diffraction (XRD) measurements in three different types of pressure cells, we have established the $(P, T)$ phase diagram up to pressures of $4.5 \mathrm{GPa}$. As shown in Fig. 1, we find a rapid and massive pressuredriven enhancement of the multiferroic transition temperature. Density-functional theory (DFT) calculations based on the XRD structure establish that the equally rapid rise of the two-magnon Raman energy is a consequence of the dramatic pressure-sensitivity of the $\mathrm{Cu}-\mathrm{Br}-\mathrm{Br}-\mathrm{Cu} J_{7}$ interaction, while $T_{\mathrm{N}}$ and hence $T_{\mathrm{C}}$ are driven primarily by the interplane coupling (most strongly by $J_{2}$ ). There is no evidence for saturation of this behavior up to the largest pressures studied, where the material remains highly insulating, confirming that there is plenty of room at the top for pressure tuning of $T_{\mathrm{C}}$ in $\mathrm{CuBr}_{2}$.

The structure of this article is as follows: In Sec. II we present details of our sample and experimental methods and summarize our theoretical analysis. In Sec. III we present the results of our dielectric measurements in two different types of pressure cell. Section IV shows analogous results for the magnetic properties obtained by NMR and Raman scattering. In Sec. V we analyze our high-pressure structural measurements by detailed first-principles calculations of electronic and magnetic energies, from which we explain the pressure evolution of all of the magnetic interactions governing the behavior of $\mathrm{CuBr}_{2}$. Section VI contains a discussion of our results, some perspective on the prospects they offer for applicable multiferroics, and a brief summary.

\section{MATERIAL AND METHODS}

Large single crystals of $\mathrm{CuBr}_{2}$ were grown by slow evaporation from aqueous solutions [29]. Because ferroelectric transitions usually cause sudden changes in the dielectric constant, we attached two copper-plate electrodes to the opposing $a b$ faces of a plate-like crystal to form a capacitor with the electric field applied perpendicular to the $a b$ plane. Measurements of the capacitance as a function of temperature $T$, pressure $P$, and magnetic field $B$ were used to indicate when a spontaneous ferroelectric polarization had developed. Dielectric measurements were performed in a piston cell (PC) using a crystal with dimensions $4 \times 1.5 \times 0.4 \mathrm{~mm}^{3}$ and in a cubic anvil cell (CAC) using a crystal with dimensions $0.7 \times 0.7 \times 0.2 \mathrm{~mm}^{3}$. The softness and propensity to chemical dissolution of the crystal meant that dielectric measurements above $2.4 \mathrm{GPa}$ were possible only in the $\mathrm{CAC}$, but not yet in anvil cells with smaller sample spaces as reported in the literature [32]; the larger error bars on the corresponding data points in Fig. 1 reflect the complexity of these measurements. The sample and copper plates were connected by using an inert epoxy and suspended in a Teflon capsule filled with Daphne oil as the pressure-transmitting medium. The pressure was calibrated at room temperature by monitoring the characteristic resistance changes of bismuth (Bi). The capacitance was measured by using an Agilent 4263B LCR meter with an excitation level of $1.0 \mathrm{~V}$ at $100 \mathrm{kHz}$.

The zero-field ${ }^{81} \mathrm{Br}(I=3 / 2)$ NMR spectra were measured by using the spin-echo method with $\pi / 2-\tau-\pi$ sequences, where $\pi / 2$ and $\pi$ denote rf pulses with respective 
time durations of 0.5 and $0.8 \mu \mathrm{s}$, and the time interval is $\tau=$ $6 \mu \mathrm{s}$. The pressure was calibrated by using the ${ }^{63} \mathrm{Cu}$ nuclear quadrupole resonance (NQR) frequency of $\mathrm{Cu}_{2} \mathrm{O}$ in the pressure cell [33]. The electronic Raman scattering measurements were performed in a confocal backscattering geometry using the $632.8 \mathrm{~nm}$ line of a He-Ne laser. The low- $T$ and high- $P$ conditions were realized by using an Almax easyLab diamond anvil cell (DAC) integrated into a Janis ST-500 optical cryostat, with argon as the pressure-transmitting medium. The pressure was calibrated by the fluorescence line of a ruby sphere loaded together with the sample inside the DAC. The high-pressure XRD experiments were performed at pressures up to $14.73 \mathrm{GPa}$ at beamline $16 \mathrm{BM}$-D of the HPCAT sector at the Advanced Photon Source (APS), Argonne National Laboratory, using a Mao-type symmetric $\mathrm{DAC}$. $\mathrm{CuBr}_{2}$ powder samples and ruby chips were loaded into the sample chamber with Neon gas as the pressure-transmission medium. Diffraction patterns were recorded on a MAR345 image plate and integrated by DIOPTAS software.

First-principles calculations of the structural and magnetic properties of $\mathrm{CuBr}_{2}$ were carried out by using densityfunctional theory with the electronic correlations for the $\mathrm{Cu}$ $3 d$ states included at the mean-field level within the generalized gradient approximation (GGA) $+U$ approach. In the first (structural) step, the lattice parameters at selected pressure values were taken from experiment and the internal positions of the $\mathrm{Br}$ ions were optimized by using the Vienna $a b$ initio simulation package (VASP) [34]. In the second (magnetic) step, the magnetic interaction parameters [Fig. 6(c)] were estimated from the structures at each pressure by computing the total magnetic energies in 27 different spin configurations using the all-electron full-potential local-orbital (FPLO) basis code [35] and then performing a total-energy mapping to a pure Heisenberg model with nine different bilinear parameters $J_{i}$ [Fig. 6(a)].

\section{HIGH-PRESSURE DIELECTRIC MEASUREMENTS}

The dielectric constant is extracted from the capacitance between two electrodes attached to the $a b$ surfaces of a single crystal, as described in Sec. II. Because the sample dimensions change under pressure, we present the capacitance rather than the dielectric constant. Results from measurements in the PC with no applied magnetic field are shown in Fig. 2(a) and in the CAC in Fig. 2(b). At $P=0.05 \mathrm{GPa}$, the capacitance at $80 \mathrm{~K}$ is $1.07 \mathrm{pF}$, which gives a dielectric constant $\varepsilon_{\mathrm{r}} \simeq 8.1$, close to the value reported previously at ambient pressure [29]. The onset of ferroelectricity is shown by a sudden increase in capacitance upon cooling below $T_{\mathrm{C}} \simeq$ $75 \mathrm{~K}$, which is slightly higher than the ambient-pressure value, $T_{\mathrm{C}}=73.5 \mathrm{~K}$. The capacitance decreases monotonically with further cooling, because of reduced charge fluctuations, and increases with rising pressure, as might be expected upon compression (reduced interlayer separations). The remarkable feature of these data is the dramatic rise of $T_{\mathrm{C}}$ to $118.5 \mathrm{~K}$ at $2.4 \mathrm{GPa}$ in the $\mathrm{PC}$, and further to $162 \mathrm{~K}$ at $4.5 \mathrm{GPa}$ in the CAC (Fig. 1). The latter $T_{\mathrm{C}}$ represents a $120 \%$ increase over the ambient-pressure value, or an average growth rate $d T_{\mathrm{C}} / d P \approx 19.7 \mathrm{~K} / \mathrm{GPa}$. Equally surprisingly, $T_{\mathrm{C}}$ continues
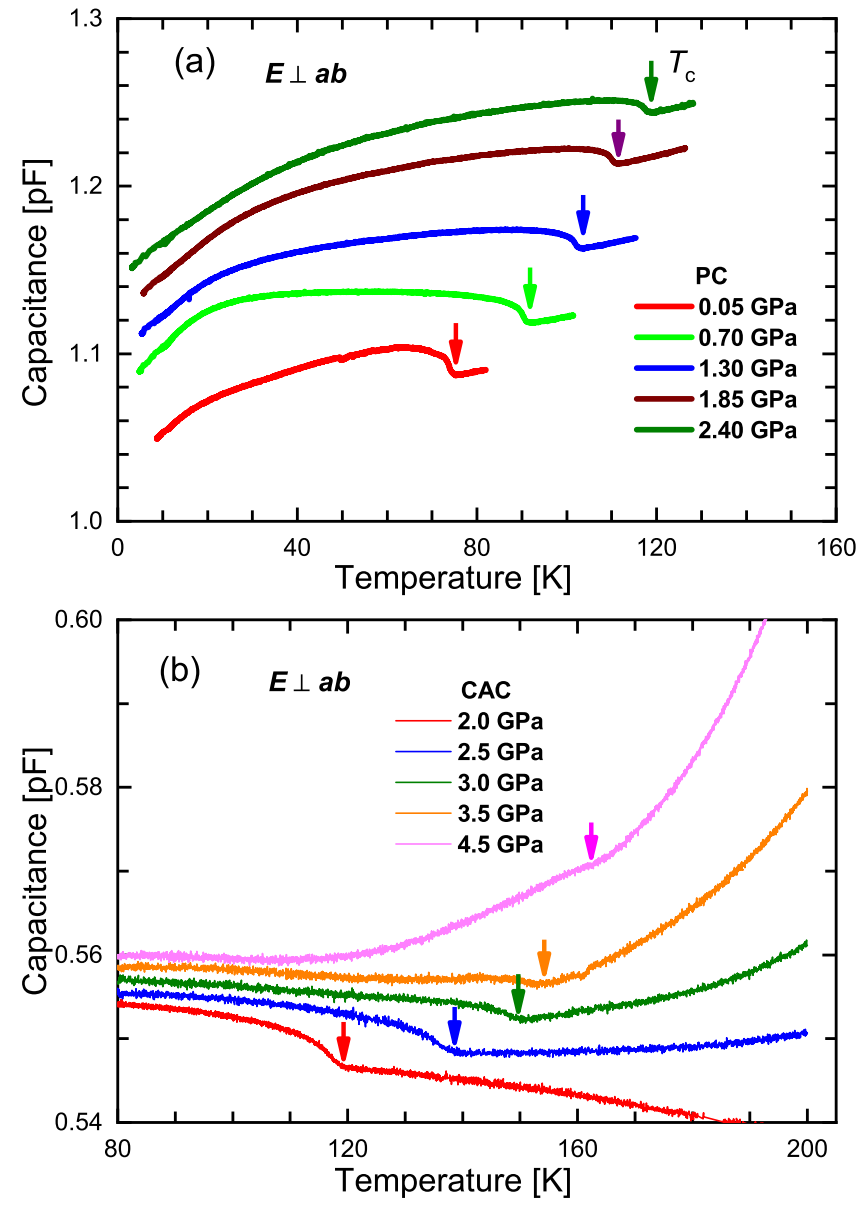

FIG. 2. Dielectric response under pressure, deduced from the capacitance between two copper plates attached to single crystals (see text), shown as a function of temperature. Measurements were performed with (a) $\mathrm{PC}$ and (b) CAC apparatus to reach pressures up to $4.5 \mathrm{GPa}$. The kink features (arrows) indicate the ferroelectric transitions.

to rise nearly linearly, with no evidence at $4.5 \mathrm{GPa}$ for a saturation of the effect.

To verify the presence of a magnetoelectric coupling, we applied an external magnetic field in the $a b$ plane in our PC measurements. This is expected to distort the spiral magnetic structure and hence to affect the ferroelectric properties. As shown in Fig. 3(a), the capacitance at $2.4 \mathrm{GPa}$ in fields $B=$ $\mu_{0} H=0,5$, and $10 \mathrm{~T}$ is constant above $T_{\mathrm{C}}=118.5 \mathrm{~K}$, and so is $T_{\mathrm{C}}$ itself. However, the magnitudes of both the capacitance and the capacitance anomaly increase monotonically with decreasing field, providing direct evidence both for a significant magnetoelectric coupling and for magnetic-orderinduced (i.e., type-II) ferroelectricity [29].

The dielectric loss $\tan \delta$ is an important figure of merit for the practical application of ferroelectric materials. In Fig. 3(b) we observe that $\tan \delta=0.013 \pm 0.001$ above $T_{\mathrm{C}}$ at all pressures reached in the PC; this value is again consistent with ambient-pressure data [29]. At all pressures, $\tan \delta$ increases weakly when the sample is cooled below $T_{\mathrm{C}}$, forming a broad low- $T$ peak whose center scales with $T_{\mathrm{C}}$. Although we do not fully understand the origin of this feature, one possibility is that the spiral spin configuration continues to fluctuate until 

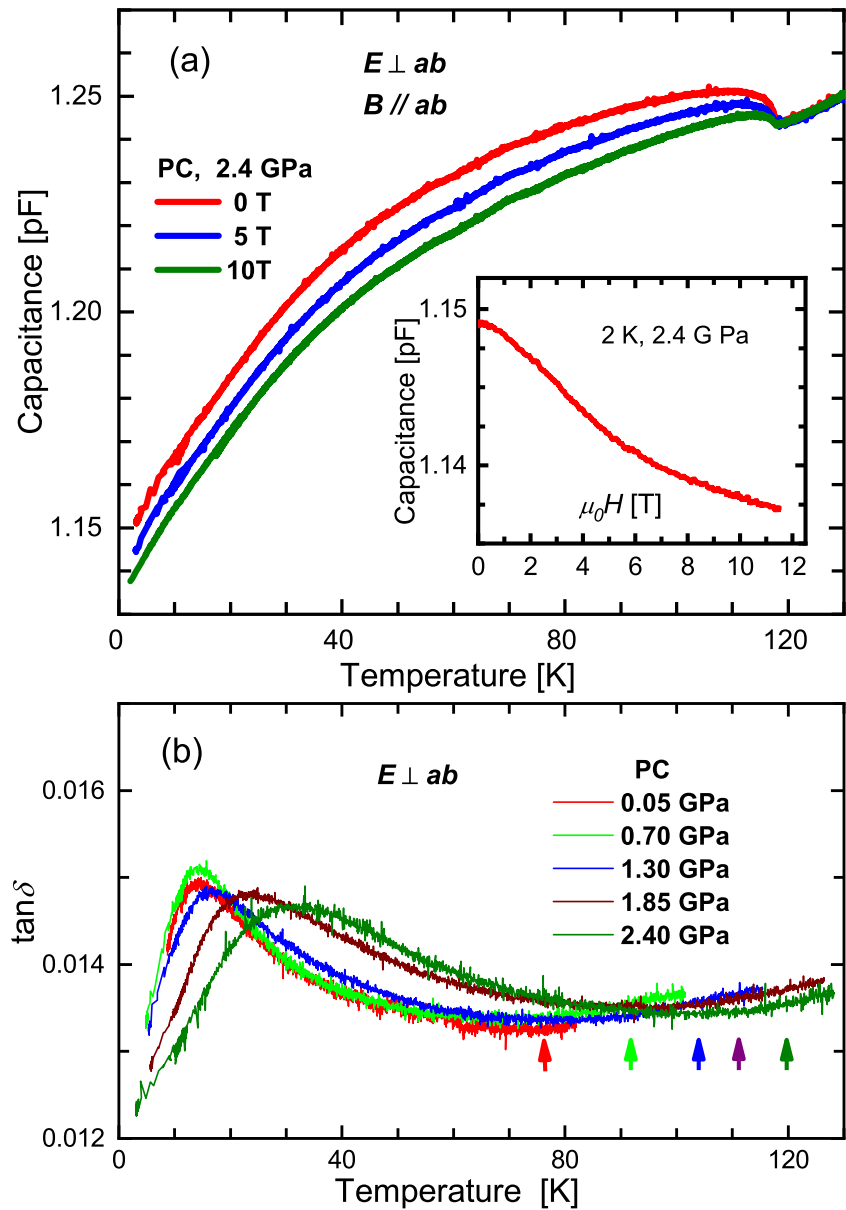

FIG. 3. Magnetoelectric coupling and dielectric loss. (a) Capacitance at $2.4 \mathrm{GPa}$, measured under an external magnetic field $B$ applied perpendicular to the electric field $E$. The inset shows the field dependence of the capacitance measured at $2 \mathrm{~K}$ and $2.4 \mathrm{GPa}$. (b) Dielectric loss, $\tan \delta$, as a function of temperature at different pressures. $\tan \delta$ shows almost no variation with the excitation level or frequency.

the sample is cooled substantially below $T_{\mathrm{C}}$, allowing for a dissipation of electrical energy into the spin system through the magnetoelectric coupling. These very small values of $\tan \delta$ at all pressures nonetheless constitute an extremely low dielectric loss, reflecting both the strongly insulating nature of $\mathrm{CuBr}_{2}$, at least up to $2.5 \mathrm{GPa}$, and the considerable potential for device applications.

\section{HIGH-PRESSURE NMR AND RAMAN SCATTERING MEASUREMENTS}

We have probed the magnetic system by zero-field NMR and Raman-scattering measurements performed over the same range of pressures as our dielectric measurements. The ${ }^{81} \mathrm{Br}$ NMR spectra at all temperatures and pressures, shown in Fig. 4(a) as the $T$-weighted spin-echo intensity as a function of frequency, have one clearly identifiable $I_{z}=1 / 2 \leftrightarrow 3 / 2$ peak [36] whose position moves systematically with both $P$ and $T$. We focus on the resonance frequency, $f(P, T)$ of this peak at each pressure and display its temperature dependence
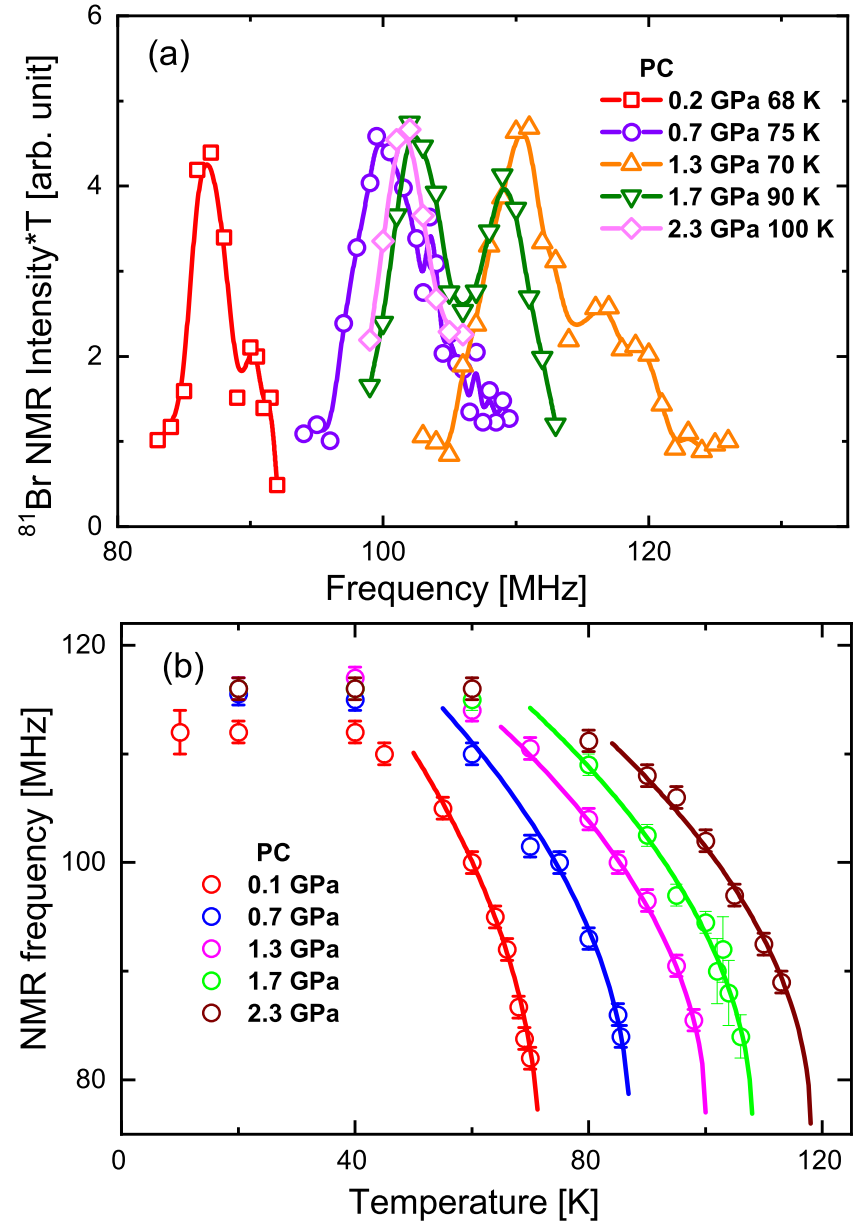

FIG. 4. NMR measurements under pressure. (a) Zero-field ${ }^{81} \mathrm{Br}$ NMR spectrum, shown in the conventional form of the product of spin-echo intensity and temperature as a function of frequency, measured in a $\mathrm{PC}$ at a selection of fixed pressures and temperatures. (b) Zero-field ${ }^{81} \mathrm{Br}$ NMR frequency $f(P, T)$, which drops sharply as the temperature approaches $T_{\mathrm{N}}(P)$; solid lines show fits to the form $f(P, T)=f_{0}\left(P, T_{\mathrm{N}}\right)+a(P)\left(T_{\mathrm{N}}-T\right)^{1 / 2}$

in Fig. 4(b). Below $T_{\mathrm{N}}, f(P, T)$ has two additive contributions, one due to the electric-field gradient (EFG), $f_{0}(P, T)$, and one from the static local hyperfine field $A_{\mathrm{hf}}\langle S\rangle$ (where $A_{\mathrm{hf}}$ is the hyperfine coupling constant and $\langle S\rangle$ is the average magnetic moment). The $f_{0}$ term is expected to change very little with temperature at low $T$, and we find it to increase only rather weakly with pressure. Because the second contribution is proportional to the magnetic order parameter, $f(P, T)$ decreases sharply as $T \rightarrow T_{\mathrm{N}}(P)$; a fit to the form $f(P, T)=$ $f_{0}(P)+a(P)\left[T_{\mathrm{N}}(P)-T\right]^{1 / 2}$ at each $P$ (solid lines) allows us to deduce the values $T_{\mathrm{N}}(P)$ up to $2.3 \mathrm{GPa}$ shown in Fig. 1 . We comment that $f(P, T)$ tends to saturate below $40 \mathrm{~K}$ [Fig. 4(b)], with $f(P, T)-f_{0}(P)=39 \mathrm{MHz}$ both at $P=0.1 \mathrm{GPa}$ and at $2.3 \mathrm{GPa}$, confirming that the ordered moment changes very little with pressure (under the assumption that $A_{\mathrm{hf}}$ does not change with $P$ ).

The Raman susceptibility is obtained by dividing the recorded scattered photon intensities by the Bose factor. Its dominant feature is the "two-magnon" excitation [37,38], which we show in Fig. 5(a) for all temperatures at a fixed 


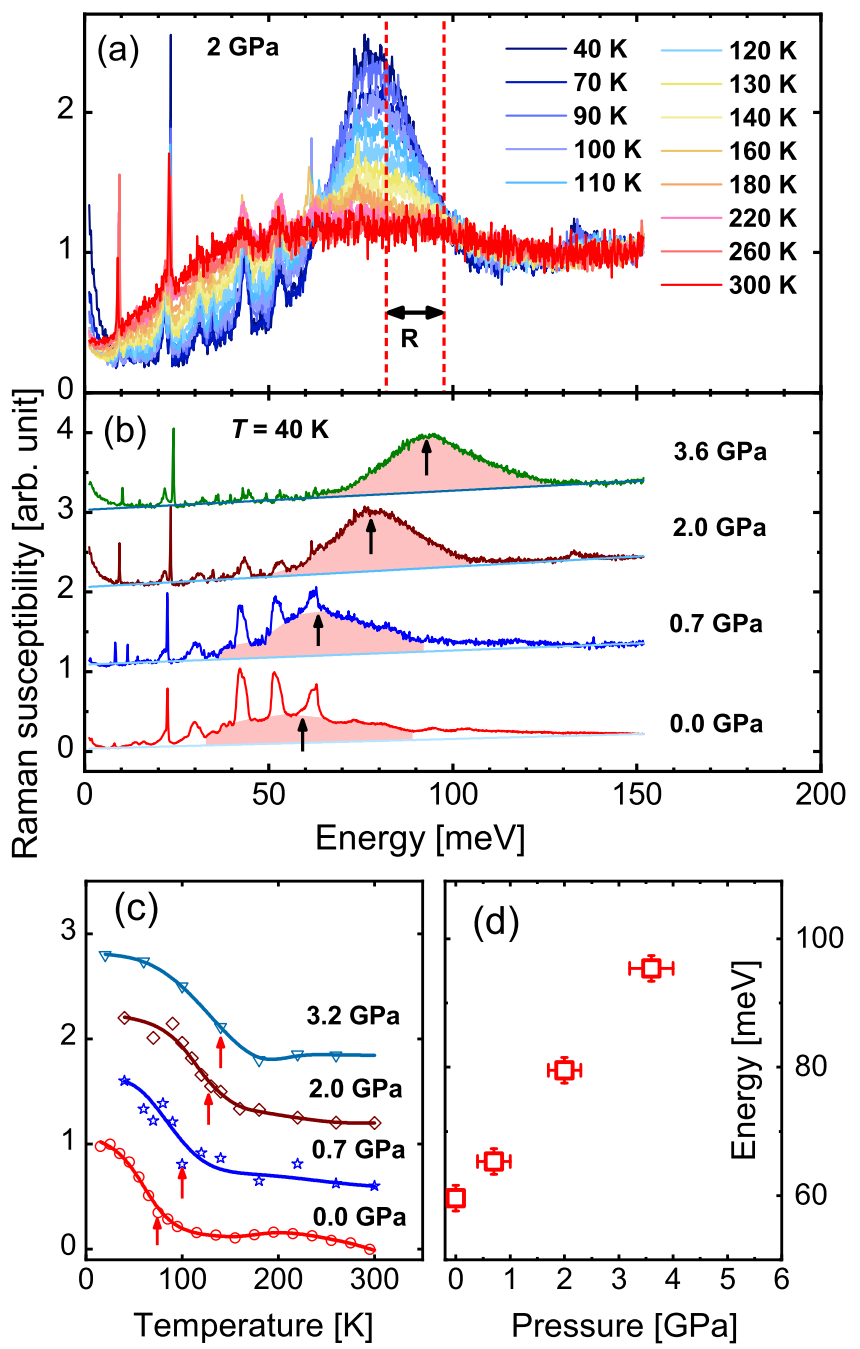

FIG. 5. Raman-scattering measurements under pressure. (a) Raman spectra measured at $2.0 \mathrm{GPa}$ over the full range of temperatures. " $\mathrm{R}$ " denotes the frequency window for the averaged Raman susceptibility. (b) Raman spectra obtained at $40 \mathrm{~K}$ under different pressures. Shaded areas indicate the two-magnon response discussed in the text and arrows indicate its characteristic central energy. Data are offset vertically for clarity. (c) Raman susceptibility averaged over an energy interval located directly above the two-magnon peak center, as shown in panel (a). Arrows indicate the approximate location of $T_{\mathrm{N}}(P)$ estimated from a $20 \%$ increase in the signal upon cooling. (d) Central two-magnon Raman energy shown as a function of pressure.

pressure of 2.0 GPa. While the sharp peaks are phonons, the two-magnon response is a very broad peak that in ordered quantum magnets bears little resemblance to the density of states of 3D spin waves [39] and persists in the paramagnetic phase due to short-range magnetic correlations. Here we observe that this broad peak sharpens at low temperatures to a form quite similar to the well-characterized cuprate response (of $\mathrm{Cu}^{2+}$ spins in a planar quantum magnet) [40]. Figure 5(b) shows this peak (shaded area) for a fixed low temperature of $40 \mathrm{~K}$ at several selected pressures. It is clear that the two-magnon energy scale increases rapidly under pressure, rising by $60 \%$ from ambient pressure to $3.6 \mathrm{GPa}$ [Fig. 5(d)].
TABLE I. Lattice parameters $a, b$, and $c$, and monoclinic angle $\beta$, obtained from the high-pressure XRD data.

\begin{tabular}{lcccc}
\hline \hline$P[\mathrm{GPa}]$ & $a[\AA]$ & $b[\AA]$ & $c[\AA]$ & $\beta\left[^{\circ}\right]$ \\
\hline 0.49 & 7.09 & 3.47 & 6.91 & 119.3 \\
1.87 & 6.95 & 3.45 & 6.75 & 118.8 \\
2.94 & 6.89 & 3.44 & 6.69 & 118.2 \\
4.15 & 6.85 & 3.44 & 6.64 & 118.3 \\
5.65 & 6.68 & 3.42 & 6.48 & 117.8 \\
6.86 & 6.60 & 3.41 & 6.43 & 117.5 \\
8.28 & 6.52 & 3.40 & 6.34 & 117.3 \\
11.12 & 6.40 & 3.38 & 6.24 & 117.0 \\
14.73 & 6.23 & 3.35 & 6.08 & 116.5 \\
\hline \hline
\end{tabular}

Despite the complexities inherent to an accurate modeling of the two-magnon response, it is safe to conclude that the relevant magnetic interactions in the system are enhanced massively by the effects of hydrostatic pressure.

A subsidiary piece of information may be extracted from the $T$-dependence of the two-magnon peak intensity, based on the empirical connection between $T_{\mathrm{N}}$ and the intensity increase upon cooling at ambient pressure [38]. In Fig. 5(c), we average the Raman susceptibility over a fixed-percentage energy range [Fig. 5(a)] located slightly above the central energy of the two-magnon peak (in order to avoid multiphonon scattering processes that overlap with the electronic signal at lower energies). This analysis allows us to extract values for $T_{\mathrm{N}}(P)$, marked by the arrows in Fig. 5(c), which again are fully consistent with the values of $T_{\mathrm{C}}(P)$ shown in Fig. 1 .

\section{HIGH-PRESSURE STRUCTURAL ANALYSIS AND DFT CALCULATIONS}

Our data in Figs. 2 and 3 confirm that $\mathrm{CuBr}_{2}$ retains qualitatively the same type-II multiferroic properties at all pressures below $5 \mathrm{GPa}$. To explain the giant pressure-sensitivity of $T_{\mathrm{C}}$ in a quantitative manner, we investigate the structure of $\mathrm{CuBr}_{2}$ by high-pressure XRD measurements and corresponding DFT calculations. XRD was performed up to $15 \mathrm{GPa}$ using synchrotron radiation at the Advanced Photon Source, as outlined in Sec. II, and we quote the lattice parameters over the full pressure range in Table I. The key features of our results are first that the monoclinic structure is preserved for all pressures and second that, as represented graphically in Fig. 6(b), and as may be expected to lowest order, the chain units remain rather rigid. There are only small relative changes to the $b$-axis dimension and the angle $\beta$, whereas the $a$ and $c$ lattice parameters, which correspond to the chains being compressed together, change by approximately $12 \%$. We comment that, although one might expect the ionic displacement associated with the ferroelectric transition (inset Fig. 1) to lift the symmetry and interfere with the magnetic interactions, this value turns out to be truly vanishingly small (from the pyroelectric current [29] one may estimate it to be $0.4 \mathrm{fm}$ on each $\mathrm{Br}^{-}$ion) and hence plays no role in the structure or magnetism of the low- $T$ phase.

Our first-principles calculations under pressure are a twostep process, as described in Sec. II. First we perform a structural optimization at selected experimental pressures by 

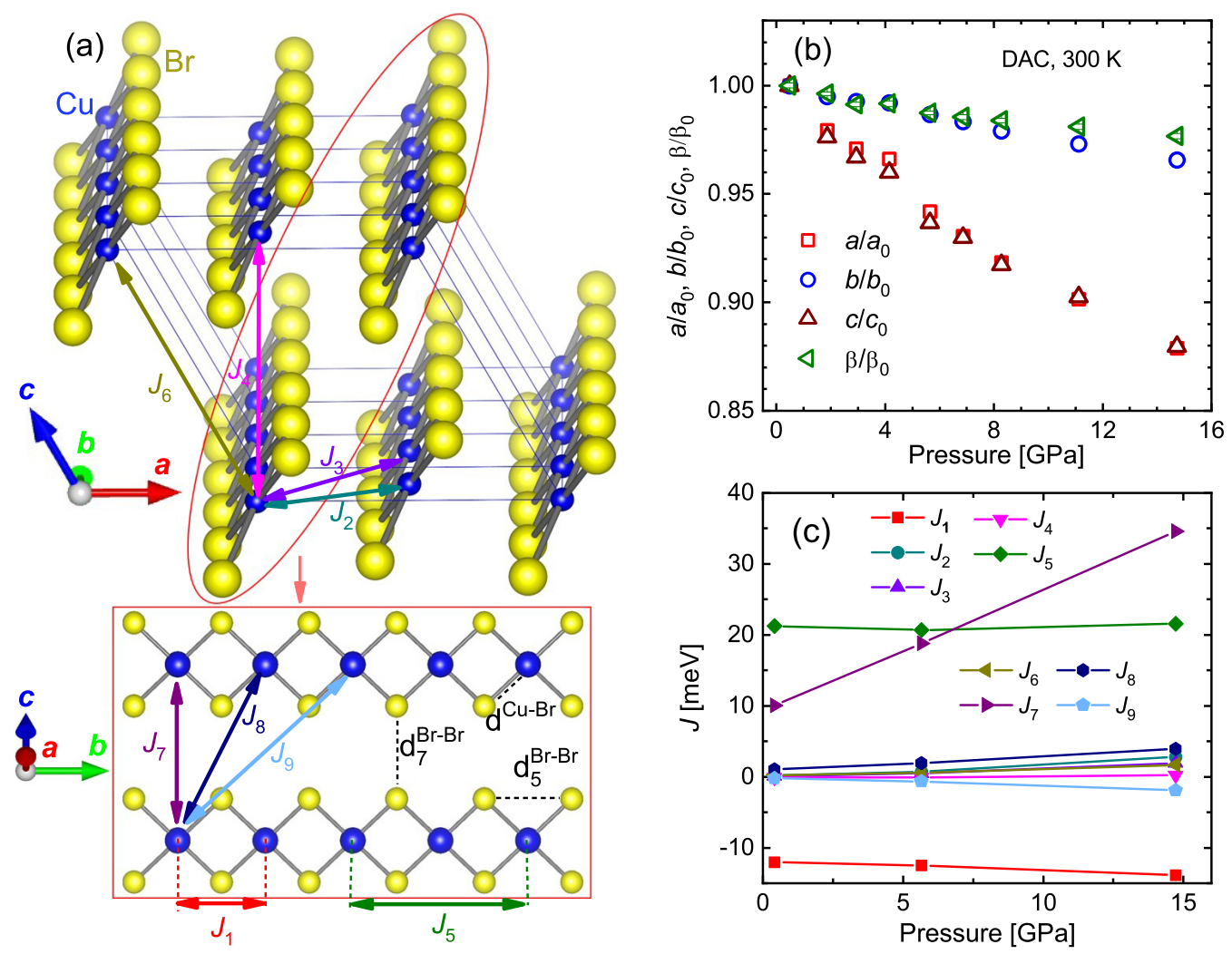

FIG. 6. Crystal structure and magnetic interactions under pressure. (a) Crystal structure of $\mathrm{CuBr}_{2}$ viewed nearly parallel to the $b$ axis. The inset displays the structure of two chains lying almost precisely in the $b(a+c)$ plane. The nine $\mathrm{Cu}-\mathrm{Cu}$ superexchange interactions computed under pressure are indicated. (b) Relative change of the lattice parameters $a, b$, and $c$, as well as of the angle $\beta$, determined by XRD at $300 \mathrm{~K}$ in a $\mathrm{DAC}$ and shown as functions of pressure. The lattice remains in a monoclinic structure at all pressures. The DAC measurements are normalized to the base-pressure dimensions obtained at $P=0.49 \mathrm{GPa}, a_{0}=7.09 \AA, b_{0}=3.47 \AA, c_{0}=6.91 \AA$, and $\beta_{0}=119.3^{\circ}$. (c) Pressure-dependence of the magnetic interactions $J_{i}$ deduced from the DFT calculations.

fixing the lattice parameters to those of the corresponding XRD measurements and relaxing the internal ionic positions within the "GGA $+U$ " approach. In this type of calculation, reliable results are obtained by using a $\vec{k}$-point mesh of size $10 \times 10 \times 10$, a plane-wave cutoff energy of $800 \mathrm{eV}$, and representative Cu-ion correlation parameters $U_{\mathrm{VASP}}=10 \mathrm{eV}$ and $J_{\mathrm{H}}=1 \mathrm{eV}$; the crystal structures are relaxed until the calculated ionic forces fall below the threshold $10^{-3} \mathrm{eV} / \AA$. For correlated systems, it is in general necessary to include the spin degrees of freedom of the transition-metal cation to ensure reliable structural predictions that, however, are quite insensitive to the actual magnetic order; here a FM order was imposed, which resulted in a total magnetic moment of $1.0 \mu_{\mathrm{B}}$ per formula unit.

For the second step of interpreting the magnetic properties of $\mathrm{CuBr}_{2}$, we identify $i=9$ significant superexchange paths, $\left\{J_{i}\right\}$, as shown in Fig. 6(a). These span the three spatial dimensions of the system and their values can be expected to determine the physics of the $b$-axis chains, the $b(a+c)$ planes, and 3D magnetic ordering. We then compute the total energies of 27 different magnetic configurations and map these to a Heisenberg model with interaction parameters $J_{i}$. The magnetic-energy calculations used two different supercells, with dimensions $2 \times 1 \times 2$ and $1 \times 4 \times 2$, and respective $k$-mesh sizes $12 \times 12 \times 12$ and $7 \times 7 \times 7$. Electronic correlations were modeled by using the GGA $+U$ functional with $U=6 \mathrm{eV}$ and $J_{\mathrm{H}}=1 \mathrm{eV}$; it is this (FPLO) value of $U$ which has a direct influence on the energy scale of the magnetic interactions. The fit to the spin model was performed by a least-squares regression analysis of the overdetermined system of 27 equations with 11 unknowns (nine superexchange parameters and two nonmagnetic contributions to the total energy, one for each supercell). Calculated $J_{i}$ values for three representative pressures taken from the XRD study, namely $0.49,5.65$, and $14.73 \mathrm{GPa}$, are shown in Fig. 6(c). The mean-square total-energy deviation between the $a b$ initio calculation and the spin model was $0.06 \mathrm{meV} / \mathrm{Cu}$ for $P=$ $0.49 \mathrm{GPa}, 0.16 \mathrm{meV} / \mathrm{Cu}$ for $P=5.65 \mathrm{GPa}$, and $0.52 \mathrm{meV} / \mathrm{Cu}$ for $P=14.73 \mathrm{GPa}$, indicating the reliability of the spin model at all pressures.

Considering first the chain units, clearly $J_{1}$ and $J_{5}$ change rather little with pressure, which to lowest order may be expected from the small changes to the $b$-axis lattice parameter [Fig. 6(b)]. In more detail, the FM [41] $\mathrm{Cu}-\mathrm{Br}-\mathrm{Cu} J_{1}$ interaction is often very sensitive to the bond angle, but here this is found to change by less than $0.5^{\circ}$ in a regime close to its optimal value [42]. While the $\mathrm{AF} J_{5}$ bond is a $\mathrm{Cu}-\mathrm{Br}-\mathrm{Br}-\mathrm{Cu}$ "super-superexchange" path that also depends on the bond angle, this remains largely fixed by the rigidity of the chains.

The dominant physics of the system occurs in the $b(a+c)$ plane due to $J_{7}$, which increases from 10 to $18 \mathrm{meV}$ up to $5.65 \mathrm{GPa}$ and then to $35 \mathrm{meV}$ at $14.73 \mathrm{GPa}$. This giant 
TABLE II. Cu-Br distance, $d^{\mathrm{Cu}-\mathrm{Br}}$, and $\mathrm{Br}-\mathrm{Br}$ distances, respectively $d_{5}^{\mathrm{Br}-\mathrm{Br}}$ and $d_{7}^{\mathrm{Br}-\mathrm{Br}}$ for the $J_{5}$ and $J_{7}$ paths represented in Fig. 6(a), computed by DFT for pressures of $0.49,5.65$, and $14.73 \mathrm{GPa}$.

\begin{tabular}{lccc}
\hline \hline$P[\mathrm{GPa}]$ & $d^{\mathrm{Cu}-\mathrm{Br}}[\AA]$ & $d_{5}^{\mathrm{Br}-\mathrm{Br}}[\AA]$ & $d_{7}^{\mathrm{Br}-\mathrm{Br}}[\AA]$ \\
\hline 0.49 & 2.440 & 3.468 & 3.651 \\
5.65 & 2.421 & 3.423 & 3.380 \\
14.73 & 2.375 & 3.350 & 3.123 \\
\hline \hline
\end{tabular}

enhancement actually changes the nature of the planar magnetism from $b$-axis dominated at ambient pressure to spatially isotropic at $5 \mathrm{GPa}$ to $(a+c)$-axis dominated at $15 \mathrm{GPa}$; however, in the absence of significant frustration it has no effect on the $b$-axis spiral order. The huge rise of $J_{7}$ under pressure may be understood completely from the fact that it is also a $\mathrm{Cu}-\mathrm{Br}-\mathrm{Br}-\mathrm{Cu}$ path, with the same geometry as $J_{5}$ [Fig. 6(a)], and while the $\mathrm{Cu}-\mathrm{Br}$ distance and angle are strongly constrained in the chain units, the $\mathrm{Br}-\mathrm{Br}$ bond in the $(a+c)$ direction takes up most of the unit-cell compression. As Table II makes clear, it shrinks from being $0.18 \AA$ longer than the comparable distance in $J_{5}$ at $0.49 \mathrm{GPa}$ to $0.23 \AA$ shorter at $14.73 \mathrm{GPa}$.

Physically, these three interactions create the dominant energy scales in the magnon dispersion, and $J_{7}$ would account completely for the rapid pressure enhancement observed in the two-magnon Raman signal [Figs. 5(b) and 5(d)]. Our absolute parameter values are controlled by the effective $U$ in the calculations, but in $\mathrm{CuBr}_{2}$ it is difficult to obtain an experimental benchmark due to sample decomposition issues in the measurement of the high-temperature susceptibility [29] and theoretical issues in interpreting the two-magnon Raman energy (Sec. IV). Thus we do not attempt to fit $U$ and simply use a value $(6 \mathrm{eV})$ typical for insulating inorganic $\mathrm{Cu}$ systems. Still, $J_{1}, J_{5}$, and $J_{7}$ span only two spatial dimensions and to discuss the $3 \mathrm{D}$ magnetic order it is necessary to consider the interplane interactions. We find that the second-shortest path in the system, $J_{2}$, which creates a zigzag interchain network in the $a b$ plane, also rises by a factor of five from 0.49 to $5.65 \mathrm{GPa}$ (and a further factor of four to $14.73 \mathrm{GPa}$ ). Similar rises can also be found in the slightly weaker $J_{3}$ and $J_{6}$ interactions. These results, which are easy to justify by considering the pressure-induced changes to interchain spin density in the $a b$ and $b c$ planes, account for the steep rise in $T_{\mathrm{N}}$, and hence in $T_{\mathrm{C}}$, over the pressure range of Fig. 1. It is clear from our XRD measurements and DFT calculations that this $T_{\mathrm{C}}$ enhancement can continue to far higher pressures, where $J_{7}$ will also play an increasing role in raising $T_{\mathrm{N}}$, with no intervening structural transition. These results raise the prospect of room-temperature multiferroicity in suitably strained $\mathrm{CuBr}_{2}$.

\section{DISCUSSION AND CONCLUSION}

Figure 1 shows that the two intrinsically linked characteristic temperatures $T_{\mathrm{C}}$ and $T_{\mathrm{N}}$, as measured by a range of probes and in a number of different pressure cells, rise strongly with pressure. Figure 5(d) shows a proportionally similar and equally quasilinear rise in the central energy scale determined by two-magnon Raman scattering. To our knowledge, our maximal $T_{\mathrm{C}}$ of $162 \mathrm{~K}$, achieved at $4.5 \mathrm{GPa}$ in a CAC, is unprecedentedly high for a nonoxide type-II multiferroic. Furthermore, although it remains below that of some oxide type-II multiferroics, such as $\mathrm{CuO}$ and $\mathrm{YBaCuFeO}_{5}\left(T_{\mathrm{C}} \approx\right.$ $230 \mathrm{~K}[28,43])$, many of these suffer from higher dielectric loss due to their semiconducting nature $[28,44,45]$. The persistence of low dielectric loss in $\mathrm{CuBr}_{2}$ under pressure, despite the increase in orbital hybridization that should move the system towards metallicity, constitutes a major advantage for electronic applications.

We stress that the characteristic magnetic energy scales in $\mathrm{CuBr}_{2}$, reflected in the energy of the two-magnon peak, are much higher than $T_{\mathrm{N}}$. This indicates that both frustration and dimensionality effects play a strong role in suppressing $T_{\mathrm{N}}$ at ambient pressure, and that the effect of pressure is to reduce both. Indeed, our DFT calculations demonstrate that the primary change is caused by the interchain $(a+c)$ axis coupling $J_{7}$, which enhances the two-dimensional (2D) nature and makes chain frustration less energetically relevant. This said, it is important to note that neither the rising $J_{7}$ nor any of the other pressure-enhanced interactions has a significant effect on the existence of the in-chain frustration, which creates the helical $b$-axis spin state required for type-II multiferroicity. Beyond $J_{7}$, we have shown that the interchain $a b$-plane coupling $J_{2}$ plays the leading role in making the system 3D and hence governs the value of $T_{\mathrm{N}}$. Despite being very low at ambient pressure, its high pressure-sensitivity causes the strong rise of $T_{\mathrm{N}}$ whose lower end we have characterized in the present work. We comment that such massive pressure effects on magnetism are known in $\mathrm{Cu}$-based metalorganic materials [46], due to a combination of soft structures and highly directional ligand paths, but are uncommon in inorganic $\mathrm{Cu}$ systems and, to date, unknown in multiferroic systems.

In summary, we have demonstrated how strongly the magnetic interactions in $\mathrm{CuBr}_{2}$ are changed by pressure, and how this makes it possible to effect a giant enhancement of the multiferroic $T_{\mathrm{C}}$ by using any available methods for structural control. Dielectric investigations of $\mathrm{CuBr}_{2}$ at pressures higher than our current limit of $4.5 \mathrm{GPa}$ are certainly required. Alternatively, different methods of structural tuning, including chemical pressure [47] and epitaxial stress [48], also affect the magnetism of low-dimensional systems in ways similar to a hydrostatic pressure. Thin-film growth with epitaxial stress applied along the $a$ or $c$ direction, by the choice of a suitable substrate, should be a particularly valuable route to higher $T_{\mathrm{C}}$ values in $\mathrm{CuBr}_{2}$. We conclude by stressing once again that the pressures we have investigated remain far from saturating the $T_{\mathrm{C}}$ increase in $\mathrm{CuBr}_{2}$, and that they seem not to impair any of the significant magnetoelectric coupling, the dielectric loss, or the insulating properties of the material, all of which present major technical advantages for application purposes.

\section{ACKNOWLEDGMENTS}

We thank R. Kremer for helpful discussions. Work at Renmin University of China was supported by the Ministry of Science and Technology of China (MOSTC) under Grant No. 2016YFA0300504, the National Natural Science Foundation of China (NSFC) under Grant No. 51872328 and the 
Fundamental Research Funds for the Central Universities and the Research Funds of Renmin University of China under Grant No. 15XNLQ07. Work at Peking University was supported by the NSFC (Grants No. 11888101, No. 11874069, and No. 11725415) and the National Basic Research Program of China under Grant No. 2018YFA0305600. J.G.C. is supported by the MOSTC (Grant No. 2018YFA0305700), NSFC (Grants No. 11834016 and No. 11874400) and the Chinese Academy of Sciences (Grants No. XDB25000000 and No. QYZDB-SSW-SLH013). The XRD measurements were performed at sector 16 BM-D (HPCAT) of the Advanced Photon Source, a U.S. Department of Energy (DoE) Office of Science user facility operated by Argonne National Laboratory (ANL) under Contract No. DE-AC02-06CH11357. HPCAT operations were supported by the DoE-NNSA under Award No. DE-NA0001974, with partial instrumentation funding by the NSF. A $b$ initio calculations were supported by the Deutsche Forschungsgemeinschaft (DFG) through Grant No. VA117/15-1 with computer time provided by the Centre for Scientific Computing (CSC) in Frankfurt.
[1] N. A. Spaldin and M. Fiebig, The renaissance of magnetoelectric multiferroics, Science 309, 391 (2005).

[2] R. Ramesh and N. A. Spaldin, Multiferroics: Progress and prospects in thin films, Nat. Mater. 6, 21 (2007).

[3] N. A. Spaldin, S.-W. Cheong, and R. Ramesh, Multiferroics: Past, present, and future, Phys. Today 63(10), 38 (2010).

[4] J. Ma, J. M. Hu, Z. Li, and C. W. Nan, Recent progress in multiferroic magnetoelectric composites: From bulk to thin films, Adv. Mater. 23, 1062 (2011).

[5] H. J. Zhao, W. Ren, Y. R. Yang, J. Iniguez, X. M. Chen, and L. Bellaiche, Near room-temperature multiferroic materials with tunable ferromagnetic and electrical properties, Nat. Commun. 5, 4021 (2014).

[6] N. Ortega, A. Kumar, J. F. Scott, and R. S. Katiyar, Multifunctional magnetoelectric materials for device applications, J. Phys.: Condens. Matter 27, 504002 (2015).

[7] M. Fiebig, T. Lottermoser, D. Meier, and M. Trassin, The evolution of multiferroics, Nat. Rev. Mater. 1, 16046 (2016).

[8] L. Seixas, A. S. Rodin, A. Carvalho, and A. H. Castro Neto, Multiferroic Two-Dimensional Materials, Phys. Rev. Lett. 116, 206803 (2016).

[9] C. X. Huang, Y. P. Du, H. P. Wu, H. J. Xiang, K. M. Deng, and E. J. Kan, Prediction of Intrinsic Ferromagnetic Ferroelectricity in a Transition-Metal Halide Monolayer, Phys. Rev. Lett. 120, 147601 (2018).

[10] D. Khomskii, Classifying multiferroics: Mechanisms and effects, Physics 2, 20 (2009).

[11] G. Catalan and J. F. Scott, Physics and applications of bismuth ferrite, Adv. Mater. 21, 2463 (2009).

[12] P. Jain, A. Stroppa, D. Nabok, A. Marino, A. Rubano, D. Paparo, M. Matsubara, H. Nakotte, M. Fiebig, S. Picozzi, E. S. Choi, A. K. Cheetham, C. Draxl, N. S. Dalal, and V. S. Zapf, Switchable electric polarization and ferroelectric domains in a metal-organic-framework, npj Quantum Mater. 1, 16012 (2016).

[13] T. Kimura, T. Goto, H. Shintani, K. Ishizaka, Y. Arima, and Y. Tokura, Magnetic control of ferroelectric polarization, Nature (London) 426, 55 (2003).

[14] S.-W. Cheong and M. Mostovoy, Multiferroics: A magnetic twist for ferroelectricity, Nat. Mater. 6, 13 (2007).

[15] J. J. Zhang, L. F. Lin, Y. Zhang, M. H. Wu, B. I. Yakobson, and S. Dong, Type-II multiferroic $\mathrm{Hf}_{2} \mathrm{VC}_{2} \mathrm{~F}_{2}$ MXene monolayer with high transition temperature, J. Am. Chem. Soc. 140, 9768 (2018).

[16] Y. Tokura and S. Seki, Multiferroics with spiral spin orders, Adv. Mater. 22, 1554 (2010).
[17] H. Katsura, N. Nagaosa, and A. V. Balatsky, Spin Current and Magnetoelectric Effect in Noncollinear Magnets, Phys. Rev. Lett. 95, 057205 (2005).

[18] I. A. Sergienko and E. Dagotto, Role of the DzyaloshinskiiMoriya interaction in multiferroic perovskites, Phys. Rev. B 73, 094434 (2006).

[19] M. Mostovoy, Ferroelectricity in Spiral Magnets, Phys. Rev. Lett. 96, 067601 (2006).

[20] S. Dong, R. Yu, S. Yunoki, J.-M. Liu, and E. Dagotto, Origin of multiferroic spiral spin order in the $R \mathrm{MnO}_{3}$ perovskites, Phys. Rev. B 78, 155121 (2008).

[21] H.-J. Xiang, S.-H. Wei, M.-H. Whangbo, and J. L. F. Da Silva, Spin-Orbit Coupling and Ion Displacements in Multiferroic $\mathrm{TbMnO}_{3}$, Phys. Rev. Lett. 101, 037209 (2008).

[22] R. Haumont, P. Bouvier, A. Pashkin, K. Rabia, S. Frank, B. Dkhil, W. A. Crichton, C. A. Kuntscher, and J. Kreisel, Effect of high pressure on multiferroic $\mathrm{BiFeO}_{3}$, Phys. Rev. B 79, 184110 (2009).

[23] T. Varga, A. Kumar, E. Vlahos, S. Denev, M. Park, S. Hong, T. Sanehira, Y. Wang, C. J. Fennie, S. K. Streiffer, X. Ke, P. Schiffer, V. Gopalan, and J. F. Mitchell, Coexistence of Weak Ferromagnetism and Ferroelectricity in the High Pressure $\mathrm{LiNbO}_{3}$-Type Phase of $\mathrm{FeTiO}_{3}$, Phys. Rev. Lett. 103, 047601 (2009).

[24] J. Ruiz-Fuertes, S. Lopez-Moreno, J. Lopez-Solano, D. Errandonea, A. Segura, R. Lacomba-Perales, A. Munoz, S. Radescu, P. Rodriguez-Hernandez, M. Gospodinov, L. L. Nagornaya, and C. Y. Tu, Pressure effects on the electronic and optical properties of $\mathrm{AWO}_{4}$ wolframites $(A=\mathrm{Cd}, \mathrm{Mg}$, $\mathrm{Mn}$, and $\mathrm{Zn}$ ): The distinctive behavior of multiferroic $\mathrm{MnWO}_{4}$, Phys. Rev. B 86, 125202 (2012).

[25] X. Rocquefelte, K. Schwarz, P. Blaha, S. Kumar, and J. van den Brink, Room-temperature spin-spiral multiferroicity in high-pressure cupric oxide, Nat. Commun. 4, 2511 (2013).

[26] T. Aoyama, K. Yamauchi, A. Iyama, S. Picozzi, K. Shimizu, and T. Kimura, Giant spin-driven ferroelectric polarization in $\mathrm{TbMnO}_{3}$ under high pressure, Nat. Commun. 5, 4927 (2014).

[27] P. A. Lee, N. Nagaosa, and X.-G. Wen, Doping a Mott insulator: Physics of high-temperature superconductivity, Rev. Mod. Phys. 78, 17 (2006).

[28] T. Kimura, Y. Sekio, H. Nakamura, T. Siegrist, and A. P. Ramirez, Cupric oxide as an induced-multiferroic with high- $T_{\mathrm{c}}$, Nat. Mater. 7, 291 (2008). 
[29] L. Zhao, T.-L. Hung, C.-C. Li, Y.-Y. Chen, M.-K. Wu, R. K. Kremer, M. G. Banks, A. Simon, M.-H. Whangbo, C. Lee, J. S. Kim, I. Kim, and K. H. Kim, $\mathrm{CuBr}_{2}$ - A new multiferroic material with high critical temperature, Adv. Mater. 24, 2469 (2012).

[30] C. Lee, J. Liu, H.-J. Koo, M.-H. Whangbo, R. Kremer, and A. Simon, Investigation of the spin exchange interactions and the magnetic structure of the high-temperature multiferroic $\mathrm{CuBr}_{2}$, Phys. Rev. B 86, 060407 (2012).

[31] S. Seki, T. Kurumaji, S. Ishiwata, H. Matsui, H. Murakawa, Y. Tokunaga, Y. Kaneko, T. Hasegawa, and Y. Tokura, Cupric chloride $\mathrm{CuCl}_{2}$ as an $S=1 / 2$ chain multiferroic, Phys. Rev. B 82, 064424 (2010).

[32] T. Honda, T. Aoyama, J. S. White, Th. Strässle, L. Keller, M. Kenzelmann, F. Honda, A. Miyake, K. Shimizu, Y. Wakabayashi, and T. Kimura, Pressure effect on magnetism and multiferroicity in $\mathrm{Mn}_{2} \mathrm{GeO}_{4}$, Phys. Rev. B 89, 104405 (2014).

[33] A. P. Reyes, E. T. Ahrens, R. H. Heffner, P. C. Hammel, and J. D. Thompson, Cuprous oxide manometer for high-pressure magnetic resonance experiments, Rev. Sci. Instrum. 63, 3120 (1992).

[34] G. Kresse and J. Furthmüller, Efficient iterative schemes for $a b$ initio total-energy calculations using a plane-wave basis set, Phys. Rev. B 54, 11169 (1996).

[35] K. Koepernik and H. Eschrig, Full-potential nonorthogonal local-orbital minimum-basis band-structure scheme, Phys. Rev. B 59, 1743 (1999).

[36] R. Wang, J. Zheng, T. Chen, P. Wang, J. Zhang, Y. Cui, C. Wang, Y. Li, S. Xu, F. Yuan, and W. Yu, NMR evidence of charge fluctuations in multiferroic $\mathrm{CuBr}_{2}$, Chin. Phys. B 27, 037502 (2018).

[37] P. A. Fleury and R. Loudon, Scattering of light by one- and two-magnon excitations, Phys. Rev. 166, 514 (1968).

[38] C. Wang, D. Yu, X. Liu, R. Chen, X. Du, B. Hu, L. Wang, K. Iida, K. Kamazawa, S. Wakimoto, J. Feng, N. Wang, and Y. Li, Direct observation of magnetoelastic excitations in a quasi-1D spiral magnet, Phys. Rev. B 96, 085111 (2017).
[39] T. P. Devereaux and R. Hackl, Inelastic light scattering from correlated electrons, Rev. Mod. Phys. 79, 175 (2007).

[40] N. Chelwani, A. Baum, T. Böhm, M. Opel, F. Venturini, L. Tassini, A. Erb, H. Berger, L. Forró, and R. Hackl, Magnetic excitations and amplitude fluctuations in insulating cuprates, Phys. Rev. B 97, 024407 (2018).

[41] J. Kanamori, Superexchange interaction and symmetry properties of electron orbitals, J. Phys. Chem. Solids 10, 87 (1959).

[42] O. Oeckler and A. Simon, Redetermination of the crystal structure of copper dibromide, $\mathrm{CuBr}_{2}$, Z. Kristallogr. 215, 13 (2000).

[43] M. Morin, A. Scaramucci, M. Bartkowiak, E. Pomjakushina, G. Deng, D. Sheptyakov, L. Keller, J. Rodriguez-Carvajal, N. A. Spaldin, M. Kenzelmann, K. Conder, and M. Medarde, Incommensurate magnetic structure, $\mathrm{Fe} / \mathrm{Cu}$ chemical disorder, and magnetic interactions in the high-temperature multiferroic $\mathrm{YBaCuFeO}_{5}$, Phys. Rev. B 91, 064408 (2015).

[44] T. Katsufuji, S. Mori, M. Masaki, Y. Moritomo, N. Yamamoto, and $\mathrm{H}$. Takagi, Dielectric and magnetic anomalies and spin frustration in hexagonal $R \mathrm{MnO}_{3}(R=\mathrm{Y}, \mathrm{Yb}$, and $\mathrm{Lu})$, Phys. Rev. B 64, 104419 (2001).

[45] F. Schrettle, S. Krohns, P. Lunkenheimer, J. Hemberger, N. Büttgen, H.-A. Krug von Nidda, A. V. Prokofiev, and A. Loidl, Switching the ferroelectric polarization in the $S=1 / 2$ chain cuprate $\mathrm{LiCuVO}_{4}$ by external magnetic fields, Phys. Rev. B 77, 144101 (2008).

[46] B. Wehinger, C. Fiolka, A. Lanza, R. Scatena, M. Kubus, A. Grockowiak, W. A. Coniglio, D. Graf, M. Skoulatos, J.-H. Chen, J. Gukelberger, N. Casati, O. Zaharko, P. Macchi, K. W. Krämer, S. Tozer, C. Mudry, B. Normand, and Ch. Rüegg, Giant Pressure Dependence and Dimensionality Switching in a Metal-Organic Quantum Antiferromagnet, Phys. Rev. Lett. 121, 117201 (2018).

[47] D. Jérome, The physics of organic superconductors, Science 252, 1509 (1991).

[48] P. K. Muduli, S. K. Bose, and R. C. Budhani, Stressinduced competing ferromagnetic and antiferromagnetic orders in epitaxial films of A-type antiferromagnet $\mathrm{La}_{0.45} \mathrm{Sr}_{0.55} \mathrm{MnO}_{3}$, J. Phys.: Condens. Matter 19, 226204 (2007). 